\title{
FLORÍSTICA E FITOSSOCIOLOGIA DE UM REMANESCENTE DE VEGETAÇÃO CADUCIFÓlIA ESPINHOSA ARBÓREA EM CARUARU, PERNAMBUCO ${ }^{1}$
}

\author{
Francisco Guedes Alcoforado-Filho ${ }^{2}$ \\ Everardo Valadares de Sá Barretto Sampaio ${ }^{3}$ \\ Maria Jesus Nogueira Rodal ${ }^{4}$
}

Recebido em 09/11/1999. Aceito em 15/10/2002

\begin{abstract}
RESUMO - (Florística e fitossociologia de um remanescente de vegetação caducifólia espinhosa arbórea em Caruaru, Pernambuco). A Vegetação Caducifólia Espinhosa (VCE) que recobre a maior parte do semi-árido, vulgarmente chamada caatinga, é bastante diversificada em função de diferenciações climáticas e morfopedológicas. Como pouco se sabe sobre suas características nos limites de sua distribuição, como na região fisiográfica do Agreste no planalto da Borborema, foi realizado o levantamento florístico e fitossociológico de uma área do município de Caruaru, Pernambuco. Foram amostrados todos os indivíduos com diâmetro do caule ao nível do solo $\geq 3 \mathrm{~cm}$ e altura total $\geq 1 \mathrm{~m}$, presentes em 36 parcelas de $10 \times 20 \mathrm{~m}$ (total de $0,72 \mathrm{ha}$ ). Foram coletadas 96 espécies, distribuídas por 41 famílias, com 55 espécies amostradas no levantamento fitossociológico. A maioria delas é comum a outras áreas secas do sertão nordestino, embora algumas também ocorram em ambientes com maior disponibilidade hídrica. Densidade (3.810 indivíduos ha $\left.{ }^{-1}\right)$ e área basal $\left(24,9 \mathrm{~m}^{2} \mathrm{ha}^{-1}\right)$ totais e as distribuições de diâmetros foram na faixa encontrada em outras áreas da VCE, mas o número de plantas com altura acima de $8 \mathrm{~m}$ foi maior, quase o dobro da que tinha o maior número registrado. Também as espécies mais importantes foram as mesmas encontradas em outras áreas da VCE e foram distintas das encontradas nas Florestas Estacionais de Altitude (FEA, brejos), na Vegetação Caducifólia não Espinhosa (VCNE, carrasco) e nas Florestas Ombrófilas das Terras Baixas (FOTB, Mata Atlântica), do Nordeste. Numa análise de agrupamento com base na flora, as FOTB formaram um grupo separado e no outro, VCE e FEA foram mais semelhantes que VCNE. Estruturalmente, VCE tem menos plantas de pequeno porte que FEA e VCNE e tem menos plantas com porte grande que FEA e mais que VCNE. A vegetação na área de Agreste em Caruaru é uma VCE arbórea, com porte alto e presença de algumas poucas espécies mais comuns em locais mais úmidos que o núcleo do Sertão nordestino.
\end{abstract}

Palavras-chave - Nordeste, fisionomia, vegetação caducifólia espinhosa, semi-árido, caatinga

1 Parte da Dissertação de Mestrado do primeiro Autor

2 CPAMN-EMBRAPA, Av. Duque de Caxias, 5650, CEP 64006-220, Teresina, PI, Brasil (guedes@ cpamn.embrapa.br)

3 Departamento de Energia Nuclear, Universidade Federal de Pernambuco, Av. Prof. Luís Freire, 1000, CEP 50740-540, Recife, PE, Brasil (esampaio@ufpe.br)

4 Departamento de Biologia, UFRPE. Rua Dom Manoel de Medeiros, s/n, CEP 52171-900, Recife, PE, Brasil (rodal@truenet.com.br) 


\begin{abstract}
Floristic and phytosociology of arboreal deciduous thorny vegetation remnant at Caruaru, in Pernambuco). The Deciduous Thorny Vegetation (DTV) that covers the semi-arid area of Northeastern Brazil, called caatinga, is highly diversified as a function of its climatic and morphopedological characteristics. Since little is known of its characteristics in the border of its distribution, as in the Agreste region on the Borborema plateau, a floristic and phytosociological survey was conducted in an area at Caruaru, Pernambuco. All plants $\geq 1 \mathrm{~m}$ high $\mathrm{e} \geq 3 \mathrm{~cm}$ of stem diameter within 36 plots, $10 \times 10 \mathrm{~m}$ each, had their height and diameter measured. A total of 96 species, belonging to 41 families were found in the area, of which 55 species were included in the phytosociological survey. Most of them are also found in the dry core of the Northeastern semi-arid region, but a few of them occur in places with higher water availability. Total density $\left(3,810\right.$ plant ha $\left.{ }^{-1}\right)$ and basal area $\left(24.9 \mathrm{~m}^{2} \mathrm{ha}^{-1}\right)$ and stem diameter distribution were within the range found in other DTV areas but the number of plants taller than $8 \mathrm{~m}$ was higher, almost double the previous highest value. The most important species were the same found in other DTV areas but different from those of regional Montane Forests (MF), Deciduous Non Thorny Vegetation (DNTV) and Humid Lowland Forests (HLF), in Northeast. In a cluster analysis, based on flora, HLF formed a separate group and in the other group DTV and MF were more similar than DNTV. Structurally, DTV has less small plants than both MF and DNTV and less large plants than MF but more than DNTV. The vegetation in the Agreste at Caruaru is an arboreal DTV, with tall plants and the presence of a few species more common in more humid areas than the dry core of the Northeast.
\end{abstract}

Key words - Northeast, physiognomy, deciduous thorny vegetation, semiarid, caatinga

\section{Introdução}

Diferentes sistemas de classificação da vegetação têm sido desenvolvidos para grande variedade de escalas e propósitos (MuellerDombois \& Ellenberg 1974). Mesmo considerando as diferentes abordagens, as descrições da composição, estrutura e funcionamento formam o corpo principal do conhecimento da vegetação, o componente dominante e mais acessível das comunidades ecológicas terrestres (Shimwell 1971; Kent \& Coker 1992). Diferentes autores têm observado que a fisionomia, de modo geral, reflete as condições climáticas (Sarmiento 1972). Considerando que a fisionomia representa uma característica vegetacional facilmente mensurável, tem sido tomada como eixo principal da classificação da vegetação em larga escala.

Em termos da vegetação brasileira, a classificação hierárquica ainda não está completamente delineada. Será necessário maior aporte de informações, especialmente de campo, para que sistemas atuais como o fisionômicoecológico adotado pelos técnicos do IBGE (IBGE 1992) possa ser refinado.

Dos grandes domínios florístico-vegeta- cionais brasileiros (Bigarella et al. 1975), o da caatinga nordestina é um dos mais desconhecidos. Os mapas vegetacionais atualmente disponíveis reconhecem, neste domínio, diversas tipologias destacando a Savana-estépica por sua maior extensão, especialmente nas áreas da depressão sertaneja, onde a maior parte dos indivíduos perde as folhas, como adaptação à deficiência hídrica (Kozlowski et al. 1991; Larcher 1995) e apresenta proporção significativa de espécies espinhosas. Assim, a Savana-estépica poderia ser classificada como Vegetação Caducifólia Espinhosa (VCE). Além dela, são citadas as Florestas Ombrófilas e as Florestas Estacionais nos Brejos de Altitude (FEA), e extensas faixas ecotonais, com áreas de cerrado a oeste e a mata atlântica ao sul/sudeste (IBGE 1992; Souza et al. 1994).

Por um lado, o conhecimento da flora e fisionomia da vegetação caducifólia do semi-árido tem sido bastante ampliado, especialmente nas áreas da depressão sertaneja situada na zona fisiográfica do Sertão (Tavares et al. 1969a,b; 1970; 1974b; 1975; Albuquerque et al. 1982; Lyra 1982; Santos et al. 1992; Rodal 1992; Araújo et al. 1995; Ferraz et al. 1998) e em áreas sedimentares, também no Sertão 
(Emperaire 1985; Rodal et al. 1998; Rodal et al. 1999; Figueirêdo et al. 2000), incluindo as de Vegetação Caducifólia Não Espinhosa (VCNE), localmente chamada de Carrasco (Oliveira et al. 1997; Araújo et al. 1998a,b; Araújo \& Martins 1999). Por outro lado, pouco ou quase nada se sabe a respeito da vegetação caducifólia localizada nas áreas transicionais entre as zonas fisiográficas do Sertão e da Mata, a chamada zona do Agreste. Nessas áreas ocorrem as Florestas Ombrófilas ou Estacionais, nos brejos de altitude (sensu Rodal et al. 1998), e diferentes fitofisionomias da VCE, variando de arbustiva, nas áreas mais secas, a arbórea, nas áreas com maior disponibilidade hídrica. Atualmente, não há informações que permitam distinguir claramente uma FEA de uma VCE de porte arbóreo, especialmente por falta de levantamentos quantitativos em ambas.

Para suprir parte desta lacuna, foi realizado o levantamento florístico e fitossociológico da vegetação de uma área do município de Caruaru, situada no Agreste pernambucano. O objetivo específico foi determinar as características fisionômicas e florísticas da vegetação desta área de Agreste. $\mathrm{O}$ objetivo mais amplo foi comparar estas características com as de outras áreas de VCE, de VCNE, de FEA e de Florestas Ombrófilas das Terras Baixas (FOTB, Mata Atlântica), cujos dados estivessem disponíveis na literatura. Espera-se contribuir com informações que permitam, no futuro, que se chegue a uma classificação objetiva e coerente da vegetação do semi-árido. Esta classificação deverá suportar e responder questões sobre os padrões da vegetação e os processos ambientais, o que, juntamente com as informações de espécies raras e ameaçadas de extinção, são elementos importantes para a conservação da diversidade biológica (Grossman et al. 1998).

\section{Material e métodos}

Área de estudo - O estudo foi realizado em uma área com cerca de $20 \mathrm{ha}$, pertencente à estação experimental da Empresa Pernambucana de Pesquisa Agropecuária (IPA), ( $8^{\circ} 14^{\prime} 18^{\prime \prime} \mathrm{S}, 35^{\circ} 55^{\prime} 20^{\prime \prime} \mathrm{W}, 530 \mathrm{~m}$ de altitude) no município de Caruaru, Pernambuco, que vem sendo mantida com sua cobertura vegetal nativa por pelo menos 30 anos, na zona fisiográfica do Agreste. O solo predominante é classificado como um Podzólico Amarelo tb eutrófico, abrupto, A moderado, textura franco-arenosa, fase caatinga hipoxerófila, relevo ondulado a forte ondulado. Os valores médios das determinações físicas e químicas (EMBRAPA 1997) de amostras do solo tiradas na área, na profundidade de 0 a $20 \mathrm{~cm}$, foram: areia, silte e argila, 631,229 e $140 \mathrm{~g} \mathrm{~kg}^{-1}$; umidade a 0,3 e 15 atm, 138 e $58 \mathrm{~g} \mathrm{~kg}^{-1} ; \mathrm{pH}$ em água, 5,2; $\mathrm{Na}, \mathrm{K}$, $\mathrm{Ca}, \mathrm{Mg}, \mathrm{H}$ e Al trocáveis, 6, 6, 25, 11, 43 e $3 \mu \mathrm{mol} \mathrm{kg}^{-1}$; P disponível, 2,8 $\mathrm{mg} \mathrm{kg}^{-1}$; C e N, 16,2 e $1,5 \mathrm{~g} \mathrm{~kg}^{-1}$. A temperatura média mensal varia de 19 a $23^{\circ} \mathrm{C}$ e a pluviosidade média anual é de $694 \mathrm{~mm}$, com déficit hídrico por seis meses (agosto a fevereiro).

Coleta e tratamento de dados florísticos Coletas mensais aleatórias da flora angiospérmica foram realizadas durante 18 meses, seguindo as técnicas usuais para coleta de material botânico (Bridson \& Forman 1992). A coleção foi depositado no herbário Dárdano de Andrade-Lima (IPA) da Empresa Pernambucana de Pesquisa Agropecuária. A identificação foi realizada através de morfologia comparada, usando bibliografia especializada e análise das exsicatas depositadas no herbário do Departamento de Biologia da Universidade Federal Rural de Pernambuco (PEUFR) e do IPA. As espécies foram organizadas por família no sistema de Cronquist (1988), incluindo-se informação sobre o hábito. Com objetivo de identificar a similaridade florística entre a área de estudo e diversos levantamentos realizados em tipos vegetacionais da região (Tab. 1), foi elaborada uma matriz de presença - ausência de espécies para 18 áreas citadas em 10 levantamentos: quatro áreas em Floresta Ombrófila das Terras 
Tabela 1. Coordenadas geográficas, altitude e precipitação anual média em alguns locais com levantamentos florísticos e/ou fitossociológicos, no Nordeste do Brasil.

\begin{tabular}{|c|c|c|c|c|c|}
\hline \multirow[t]{2}{*}{ Município } & \multicolumn{2}{|c|}{ Coordenadas } & \multirow{2}{*}{$\begin{array}{l}\text { Altitude } \\
\text { (m) }\end{array}$} & \multirow{2}{*}{$\begin{array}{l}\text { Precipitação } \\
(\mathrm{mm} / \mathrm{ano})\end{array}$} & \multirow[t]{2}{*}{ Referência } \\
\hline & Sul & Oeste & & & \\
\hline \multicolumn{6}{|c|}{ Vegetação Caducifólia Espinhosa (VCE) } \\
\hline Caruaru & $8^{\circ} 14^{\prime}$ & $35^{\circ} 55^{\prime}$ & $500-550$ & 612 & este trabalho \\
\hline Custódia & $8^{\circ} 06^{\prime}$ & $37^{\circ} 19^{\prime}$ & $450-470$ & 574 & Araújo et al. (1995) \\
\hline Custódia & $8^{\circ} 18^{\prime}$ & $38^{\circ} 35^{\prime}$ & $450-470$ & 650 & Rodal (1992) \\
\hline Floresta & $8^{\circ} 30^{\prime}$ & $38^{\circ} 00^{\prime}$ & $480-490$ & 631 & Rodal (1992) \\
\hline Floresta & $8^{\circ} 37^{\prime}$ & $38^{\circ} 37^{\prime}$ & $460-470$ & 631 & Rodal (1992) \\
\hline Custódia & $8^{\circ} 18^{\prime}$ & $38^{\circ} 35^{\prime}$ & $450-470$ & 650 & Rodal (1992) \\
\hline Serra Talhada & $7^{\circ} 57^{\prime}$ & $38^{\circ} 17^{\prime}$ & 500 & 639 & Ferraz (1994) \\
\hline Serra Talhada & $7^{\circ} 59^{\prime}$ & $38^{\circ} 19^{\prime}$ & 700 & 639 & Ferraz (1994) \\
\hline Floresta & $8^{\circ} 30^{\prime}$ & $38^{\circ} 00^{\prime}$ & $460-470$ & 585 & Araújo et al. (1995) \\
\hline Floresta & $8^{\circ} 37^{\prime}$ & $38^{\circ} 17^{\prime}$ & $500-530$ & 585 & Araújo et al. (1995) \\
\hline \multicolumn{6}{|c|}{ Vegetação Caducifólia Não Espinhosa (VCNE) } \\
\hline Novo Oriente & $5^{\circ} 34^{\prime}$ & $40^{\circ} 56^{\prime}$ & $760-780$ & 618 & Araújo et al. (1998a) \\
\hline Novo Oriente & $5^{\circ} 36^{\prime}$ & $40^{\circ} 54^{\prime}$ & $760-780$ & 618 & Araújo et al. (1998a) \\
\hline Novo Oriente & $5^{\circ} 32^{\prime}$ & $40^{\circ} 55^{\prime}$ & $760-770$ & 618 & Araújo et al. (1998a) \\
\hline Ubajara & $3^{\circ} 54^{\prime}$ & $40^{\circ} 59^{\prime}$ & 830 & 1100 & Araújo \& Martins (1999) \\
\hline \multicolumn{6}{|c|}{ Vegetação de transição VCE - VCNE } \\
\hline Padre Marcos & $7^{\circ} 07^{\prime}$ & $40^{\circ} 58^{\prime}$ & 420 & 637 & Oliveira et al. (1997) \\
\hline \multicolumn{6}{|c|}{ Floresta Estacional de Altitude (FEA) } \\
\hline Pesqueira & $8^{\circ} 22^{\prime}$ & $36^{\circ} 42^{\prime}$ & $860-880$ & 885 & Correia (1996) \\
\hline Jataúba & $8^{\circ} 10^{\prime}$ & $36^{\circ} 40^{\prime}$ & $1020-1120$ & 764 & Moura (1997) \\
\hline Triunfo & $7^{\circ} 49^{\prime}$ & $38^{\circ} 02^{\prime}$ & 1100 & 1230 & Ferraz (1994) \\
\hline Triunfo & $7^{\circ} 52^{\prime}$ & $38^{\circ} 17^{\prime}$ & 900 & 1000 & Ferraz (1994) \\
\hline \multicolumn{6}{|c|}{ Floresta Ombrófila de Terras Baixas (FOTB) } \\
\hline Recife & $8^{\circ} 03^{\prime}$ & $34^{\circ} 56^{\prime}$ & $50-100$ & 2243 & Guedes (1998) \\
\hline João Pessoa & $7^{\circ} 07^{\prime}$ & $34^{\circ} 53^{\prime}$ & $10-20$ & 1760 & Barbosa (1996) \\
\hline Recife & $8^{\circ} 03^{\prime}$ & $34^{\circ} 56^{\prime}$ & $30-50$ & 2243 & Lins e Silva (1996) \\
\hline Cabo & $8^{\circ} 15^{\prime}$ & $35^{\circ} 02^{\prime}$ & $20-80$ & 2143 & Siqueira et al. (2001) \\
\hline
\end{tabular}

Baixas (Barbosa 1996; Lins e Silva 1996; Guedes 1998; Siqueira et al. 2001); quatro áreas em VCE (Rodal 1992; Ferraz 1994), quatro áreas em Floresta Estacional de Altitude (Ferraz 1994; Correia 1996; Moura 1997); três áreas em Vegetação Caducifólia Não Espinhosa ou "Carrasco" (Araújo et al. 1998a) e uma área na Transição Carrasco-Caatinga de Areia (Oliveira et al. 1997). Em seguida, foi realizada uma análise de agrupamento usando o índice de similaridade de Jaccard e a técnica de ligação da média de grupo (UPGMA) O software FITOPAC 2 (Shepherd 1995) foi utilizado para realizar as análises.

Coleta e tratamento de dados quantitativos Foram demarcadas 36 parcelas, 12 ao longo de cada uma de três linhas paralelas, que cortavam transversalmente a área e que distavam $50 \mathrm{~m}$ uma da seguinte. Cada parcela tinha $10 \times 20 \mathrm{~m}$ e distava $20 \mathrm{~m}$ da seguinte. Nas parcelas, foram amostrados os indivíduos, vivos ou mortos ainda em pé, com diâmetro do caule ao nível do solo e altura totais iguais ou superiores a $3 \mathrm{~cm}$ e $1 \mathrm{~m}$, respectivamente (Rodal et al. 1992). Na análise dos dados foram calculados (Mueller-Dombois \& Ellenberg 1974) parâmetros gerais da 
comunidade (densidade total, área basal total, alturas e diâmetros médios e máximos), parâmetros relativos das espécies (densidade, frequiência, área basal, índice do valor de importância) e índice de diversidade de Shannon (Magurran 1988). Os cálculos foram feitos usando o FITOPAC 2 (Shepherd 1995).

Para comparar o porte da vegetação de áreas classificadas como VCE, incluindo a área de Agreste de Caruaru, com o de áreas de Vegetação Caducifólia Não Espinhosa e de áreas de Floresta Estacional de Altitude (Tab. 1), as densidades de indivíduos foram divididas em três classes de diâmetro do caule (3-12, $>12$ $27 \mathrm{e}>27 \mathrm{~cm})$ e uma classe de altura $(>8 \mathrm{~m})$. Os dados das áreas de VCE foram extraídos de Rodal (1992), Ferraz (1994) e Araújo et al. (1995), os de VCNE de Araújo et al. (1998a) e Araújo \& Martins (1999) e os de FEA, de Ferraz (1994), Correia (1996) e Moura (1997). Foram também incluídos os dados de uma área classificada como de transição entre VCE e VCNE (Oliveira et al. 1997). Comparações das médias de VCNE e de FEA com as médias de VCE foram feitas usando-se o teste de t para amostras independentes (Statsoft 1995).

\section{Resultados e discussão}

Florística - Nas 150 coletas realizadas foram identificados 96 táxons, dos quais 79 no nível de espécie $(82,1 \%)$, num total de 41 famílias, além de um táxon não identificado (Tab. 2). No componente arbóreo ocorreram 39 espécies, distribuídas por 19 famílias, com destaque para Euphorbiaceae e Mimosaceae, com seis e sete espécies cada, seguidas de Cactaceae, Caesalpiniaceae, Capparaceae e Rubiaceae, com três espécies cada. Em uma revisão dos levantamentos quantitativos da Vegetação Caducifólia Espinhosa (VCE) do semi-árido nordestino, Rodal (1992) observou que as quatro primeiras famílias foram as mais bem representadas.

Todas as Cactaceae coletadas em Caruaru
(Agreste) foram árvores, enquanto que em áreas do núcleo mais seco do semi-árido (Sertão) predominam espécies arbustivas e/ou herbáceas (Rodal 1992). A ocorrência de Rubiaceae no componente lenhoso dos levantamentos de VCE tem sido registrada apenas em áreas mais úmidas que este núcleo, sejam de Agreste, como Nossa Senhora da Glória e Frei Paulo (Souza 1983), ou no sopé da Chapada do Araripe, como São José do Belmonte (Tavares et al. 1969a), e Barbalha (Tavares et al. 1974b).

Os elementos arbóreos mais conspícuos da área de estudo foram: Schinopsis brasiliensis Engler (Anacardiaceae), Caesalpinia pyramidalis Tul. e Bauhinia cheilanta (Bong.) Steud. (Caesalpiniaceae) e Maprounea guianensis Aubl. (Euphorbiaceae). Eles são citados na maioria dos levantamentos da VCE (Araújo et al. 1995; Ferraz et al. 1998). Além dessas espécies deve-se registrar a presença de táxons freqüentes nas florestas nordestinas, especialmente nas Florestas de Altitude do semi-árido ou Brejos de Altitude (Sales et al. 1998), o que sugere condição de maior umidade em Caruaru, que na maioria das áreas de VCE já estudadas no semi-árido nordestino. Estes táxons foram: Alseis floribunda Schott e Randia armata (SW.) DC. (Rubiaceae), Cedrela odorata L. (Meliaceae), Clusia paralicola G. Mariz. (Clusiaceae) e Opuntia brasiliensis (Willd.) Haw. (Cactaceae).

No componente arbustivo-subarbustivo ocorreram 28 espécies, distribuídas por 14 famílias. Euphorbiaceae, com seis espécies de arbustos, e Malvaceae, com três espécies de subarbustos, foram as famílias de maior riqueza naquele componente. São espécies comuns a outras áreas da VCE, como pode ser verificado no rótulo das exsicatas do herbário IPA. Rodal (1992) observou que Euphorbiaceae apresentou o maior número de espécies entre as nanofanerófitas (Kent \& Coker 1992) em áreas da VCE menos secas, sendo substituída por Cactaceae em áreas mais secas. A alta frequiência de representantes do gênero Croton, 
Tabela 2. Lista das famílias e espécies encontradas na Vegetação Caducifólia Espinhosa da Estação Experimental do IPA, Caruaru, Pernambuco, com seu respectivo hábito. As espécies que atendiam ao critério de inclusão (A) foram separadas em amostradas nas parcelas (designadas pelo número de indivíduos amostrados) e amostráveis (A) fora das parcelas. As espécies que não atendiam ao critério de inclusão (B) foram consideradas observadas. Árvore - ARV; Arbusto - ARB; Subarbusto - SUB; Erva - ERV; cipó - CIP.

\begin{tabular}{|c|c|c|c|}
\hline Família / Espécie & Hábito & A & B \\
\hline \multicolumn{4}{|l|}{ ACANTHACEAE } \\
\hline Ruellia bahiensis (Ness) Morong & SUB & & $\mathrm{X}$ \\
\hline \multicolumn{4}{|l|}{ AMARANTHACEAE } \\
\hline Gomphrena vaga Mart. & ERV & & $\mathrm{X}$ \\
\hline \multicolumn{4}{|l|}{ ANARCARDIACEAE } \\
\hline Myracrodruon urundeuva Allemão & ARV & 36 & \\
\hline Schinopsis brasiliensis Engler & ARV & 51 & \\
\hline \multicolumn{4}{|l|}{ ARACEAE } \\
\hline Anthurium affine Schott & ERV & & $\mathrm{X}$ \\
\hline Zomicarpa riedeliana Schott & ERV & & $\mathrm{X}$ \\
\hline \multicolumn{4}{|l|}{ ASCLEPIADACEAE } \\
\hline Matelea sp. & CIP & & $\mathrm{X}$ \\
\hline \multicolumn{4}{|l|}{ ALSTROMERIACEAE } \\
\hline Bomarea salsiloides Roem & ERV & & $\mathrm{X}$ \\
\hline \multicolumn{4}{|l|}{ ASTERACEAE } \\
\hline Bidens bipinatus L. & ERV & & $\mathrm{X}$ \\
\hline Delilea biflora Kuntze & ERV & & $\mathrm{X}$ \\
\hline Melanthera latifolia (Gard.) Cabrera & ERV & & $\mathrm{X}$ \\
\hline Verbesina macrophylla (Cass.) S.F. Blake & $\mathrm{ARB}$ & & \\
\hline \multicolumn{4}{|l|}{ BEGONIACEAE } \\
\hline Begonia egleri Brade. & ERV & & $\mathrm{X}$ \\
\hline Begonia vitifolia Schott. & ARB & A & \\
\hline \multicolumn{4}{|l|}{ BIGNONIACEAE } \\
\hline Jacaranda cf. jasminoides (Thunb.) Sandw. & CIP & & $\mathrm{X}$ \\
\hline Pithecoctenium echinatum Schum. & CIP & & $\mathrm{X}$ \\
\hline BIGNONIACEAE 1 & CIP & & $\mathrm{X}$ \\
\hline \multicolumn{4}{|l|}{ BOMBACACEAE } \\
\hline Chorisia glaziovii (O. Kuntze) E. Santos & ARV & 4 & \\
\hline \multicolumn{4}{|l|}{ BORAGINACEAE } \\
\hline Cordia globosa (Jacq.) Humb., Bonpl. \& Kunth & $\mathrm{ARB}$ & 5 & \\
\hline Cordia sp. & ARV & 32 & \\
\hline Tournefortia rubicunda Salzm. & ARB & 14 & \\
\hline \multicolumn{4}{|l|}{ BROMELIACEAE } \\
\hline Bromelia karatas L. & ERV & & $\mathrm{X}$ \\
\hline Portea leptantha Harm. & ERV & & $\mathrm{X}$ \\
\hline \multicolumn{4}{|l|}{ BURSERACEAE } \\
\hline Commiphora leptophloeos (Mart.) Gillet. & ARV & 35 & \\
\hline \multicolumn{4}{|l|}{ CACTACEAE } \\
\hline Cereus jamacaru DC. & ARV & 25 & \\
\hline Opuntia brasiliensis (Willd.) Haw. & ARV & 6 & \\
\hline Pilosocereus pachicladus var. pernambucensis Zappi. & ARV & 49 & \\
\hline \multicolumn{4}{|l|}{ CAESALPINIACEAE } \\
\hline Bauhinia cheilantha (Bong.) Steud. & ARV & 245 & \\
\hline Caesalpinia pyramidalis Tul. & ARV & 308 & \\
\hline Caesalpinia leiostachya (Benth.) Ducke & ARV & 1 & \\
\hline
\end{tabular}


Tabela 2 (continuação)

Família / Espécie

\section{CAPPARACEAE}

Capparis flexuosa $\mathrm{L}$.

Capparis jacobinae Moric.

CELASTRACEAE

Maytenus rigida Mart.

CLUSIACEAE

Clusia paralicola G. Mariz

CONVOLVULACEAE

Ipomea aff. trifida G. Don

COMMELINACEAE

Commelina sp.

DIOSCOREACEAE

Dioscorea sp.

ERYTHROXYLACEAE

Erythroxylum subrotundum St. Hil.

Erythroxylum sp.

\section{EUPHORBIACEAE}

Acalypha cf. multicaulis Muell. Arg.

Cnidoscolus urens (L.) Arthur

Croton conduplicatus Kunth

Croton micans (Sw. em.) Muell. Arg.

Croton sonderianus Muell. Arg.

Croton sp.

Dalechampia sp.

Euphorbia tirucalli L.

Jatropha mollissima (Pohl.) Baill.

Manihot cf. dichotoma Ule

Maprounea guinensis Aublet

Maprounea cf. guianensis Aublet

Sapium lanceolatum (Muell. Arg.) Herber

FABACEAE

Aeschynomene mollicula Humb., Bonpl. \& Kunth.

Chaetocalyx hebecarpa Benth.

Desmodium spirale (Sw.) DC.

Dioclea grandiflora Mart. ex Benth.

Vigna peduncularis (Humb., Bonpl. \& Kunth) Fawcett \& Kendle

FLACOURTIACEAE

Casearia sp.

\section{MALPIGHIACEAE}

Heteropteris sp.

Mascagnia sepium (Jussieu) Griseb.

Ptilochaeta bahiensis Turez

\section{MALVACEAE}

Sida acuminata DC.

Sida glomerata Cav.

Wissadula contracta (Link.) R. E. Fries.

MELIACEAE

Cedrela odorata L.

MENISPERMACEAE

Cissampelos sp.
Hábito

A

B

ARV

37

ARV

16

ARV

4

ARV

11

CIP

X

ERV

X

CIP

X

ARB

2

ARB

2

ARB

2

ARB

ARB

ARB

ARB

ARB

CIP

ARV

ARV

ARV

ARV

ARV

ARV

CIP

CIP

ERV

CIP

CIP

2

1

27

78

3

A

10

14

2

187

37

ARV

3

ARB

A

CIP

ARV

106

SUB

SUB

SUB

ARV

1

CIP
X

X

X

X

X

X

X

X

$\mathrm{X}$

X

$X$
$X$
$X$
$X$


Tabela 2 (continuação)

\begin{tabular}{|c|c|c|c|}
\hline Família / Espécie & Hábito & A & $\mathrm{B}$ \\
\hline \multicolumn{4}{|l|}{ MIMOSACEAE } \\
\hline Acacia glomerosa Benth. & ARV & 7 & \\
\hline Acacia paniculata Willd. & ARV & 17 & \\
\hline Anadenanthera colubrina (Vell.) Brenam & ARV & 49 & \\
\hline Chamaecrista nictitans (L.) Moench & SUB & & $\mathrm{X}$ \\
\hline Mimosa malacocentra Mart. & $\mathrm{ARV}$ & 140 & \\
\hline Mimosa sp. & ARV & 3 & \\
\hline Piptadenia stipulacea (Benth.) Ducke & ARV & 82 & \\
\hline Pithecellobium parviflorum Benth. & ARV & 75 & \\
\hline Schrankia leptocarpa DC. & CIP & & $\mathrm{X}$ \\
\hline \multicolumn{4}{|l|}{ MYRTACEAE } \\
\hline Eugenia uvalha Camb. & ARB & 134 & \\
\hline Eugenia sp. 1 & ARV & 1 & \\
\hline Eugenia sp. 2 & $\mathrm{ARB}$ & 20 & \\
\hline MYRTACEAE 1 & ARB & 3 & \\
\hline \multicolumn{4}{|l|}{ NYCTAGINACEAE } \\
\hline Guapira noxia (Netto) Sundell & ARV & 11 & \\
\hline \multicolumn{4}{|l|}{ ORCHIDACEAE } \\
\hline Cyrtopodium aff. aliciae L. Lind. \& Rolfe & ERV & & $\mathrm{X}$ \\
\hline Vanilla chamissonis Klotzsch. & CIP & & $\mathrm{X}$ \\
\hline \multicolumn{4}{|l|}{ PLUMBAGINACEAE } \\
\hline Plumbago scandens L. & SUB & & $\mathrm{X}$ \\
\hline \multicolumn{4}{|l|}{ RHAMNACEAE } \\
\hline Gouania lupuloides (L.) Urban. & CIP & & $\mathrm{X}$ \\
\hline Ziziphus joazeiro Mart. & ARV & 2 & \\
\hline \multicolumn{4}{|l|}{ RUBIACEAE } \\
\hline Alseis floribunda Schott & ARV & 2 & \\
\hline Coutarea hexandra (Jacq.) K. Schum. & ARV & 5 & \\
\hline Randia armata (Sw.) DC. & ARV & 1 & \\
\hline \multicolumn{4}{|l|}{ SAPINDACEAE } \\
\hline Allophylus quercifolius (Mart.) Radlk. & ARV & 5 & \\
\hline Serjania glabrata Kunth. & CIP & & $\mathrm{X}$ \\
\hline \multicolumn{4}{|l|}{ SOLANACEAE } \\
\hline Solanum sp. 1 & $\mathrm{ARB}$ & 292 & \\
\hline Solanum sp. 2 & $\mathrm{ARB}$ & 7 & \\
\hline \multicolumn{4}{|l|}{ STERCULIACEAE } \\
\hline Helicteres macropetala St. Hil. & ARB & 7 & \\
\hline \multicolumn{4}{|l|}{ TILIACEAE } \\
\hline Corchorus hirtus L. & SUB & & $\mathrm{X}$ \\
\hline \multicolumn{4}{|l|}{ VERBENACEAE } \\
\hline Lantana camara $\mathrm{L}$. & ARB & 9 & \\
\hline Lippia sp. & $\mathrm{ARB}$ & 11 & \\
\hline \multicolumn{4}{|l|}{ Não identificada } \\
\hline Não identificada & ARV & 7 & \\
\hline
\end{tabular}


especialmente no subosque, confirma a importância de Euphorbiaceae na VCE, mesmo em áreas menos secas como Caruaru (Albuquerque et al. 1982; Rodal 1992; Araújo et al. 1995; Ferraz et al. 1998). Por outro lado, a presença freqüente de Eugenia uvalha Camb. é incomum em áreas de VCE.

Em relação aos cipós, foram encontradas 17 espécies distribuídas por 12 famílias, com destaque para Fabaceae e Bignoniaceae com quatro e três espécies, respectivamente (Tab. 1). Número elevado de cipós também foi encontrado por Figueirêdo et al. (2000) em uma área da VCE. Assim, ao contrário do sugerido pela literatura (Rizzini 1979), a VCE do semi-árido apresenta número considerável de espécies neste componente.

No estrato herbáceo foram registradas 13 espécies, distribuídas em nove famílias, com destaque para Asteraceae com três espécies. Anthurium affine Schott (Araceae), bastante comum nas áreas mais úmidas da VCE e nas Florestas Estacionais do Agreste pernambucano, apresentou população abundante em Caruaru. Deve-se registrar ainda o baixo número de Bromeliaceae (duas espécies), quando comparado ao de outros levantamentos florísticos de VCE (Araújo et al. 1995). Bromelia karatas L. e Portea leptantha Harm. são pouco citadas em áreas da VCE, sendo mais frequientes em Florestas Estacionais, conforme pode ser concluído pela análise dos rótulos das exsicatas.

Análise de agrupamento realizada com base em 400 táxons identificados no nível de espécie em levantamentos quantitativos em 18 áreas com diferentes tipos vegetacionais do Nordeste, incluindo a área de estudo (Fig. 1), mostrou, em primeiro nível (2\%), dois grupos distintos. O primeiro reuniu os levantamentos em Florestas Ombrófilas das Terras Baixas (Barbosa 1996; Lins e Silva 1996; Guedes 1998; Siqueira et al. 2001) e o segundo foi formado exclusivamente por levantamentos em áreas localizadas no semi-árido, englobando as seguintes formações: VCE (Rodal 1992; Ferraz 1994), FEA (Ferraz 1994; Correia 1996; Moura 1997), VCNE (Araújo et al. 1998a), transição VCE-VCNE (Oliveira et al. 1997) e a vegetação de Agreste deste trabalho.

O grupo do semi-árido foi dividido, em um segundo nível (5\%), em dois conjuntos distintos: 1) os levantamentos de VCNE e da transição Carrasco - Caatinga de Areia; e 2) os levantamentos de VCE, o deste trabalho e os das FEA. Como Rodal \& Nascimento (2002) observaram, a maior afinidade entre as Florestas Estacionais de Altitude do semi-árido de Pernambuco (Brejos de Altitude) e a VCE deve-se ao fato de as primeiras estarem localizadas no domínio do semi-árido e rodeadas por VCE, o que leva a terem espécies em comum. Muitas dessas espécies têm ampla distribuição nas Florestas Estacionais brasileiras, como Anadenanthera colubrina (Vell.) Brenam, Myracrodruon urundeuva Engler e Tabebuia impetiginosa (Mart. ex DC.) Standl. (Prado \& Gibbs 1993; Araújo \& Haridasan 1997).

Os níveis de similaridade entre os levantamentos de VCE indicam que as quatro áreas estudadas por Rodal (1992) e Ferraz (1994) formaram um grupo ao qual a área de estudo une-se com um nível de similaridade de aproximadamente $18 \%$. A maior relação do conjunto das quatro áreas e a menor similaridade desse conjunto com á área de estudo deve-se aos seguintes aspectos: a) as quatro áreas apresentaram mais espécies em comum; b) cada uma dessas quatro áreas tem, individualmente, menos espécies que a área de estudo; c) na área de estudo ocorrem espécies comuns aos demais levantamentos quantitativos de VCE e outras raramente presentes naquelas quatro áreas. Dentre essas últimas espécies destacam-se: Eugenia uvalha Camb., Clusia paralicola G. Mariz, Opuntia brasiliensis (Willd.) Haw., Coutarea hexandra (Jacq.) K. Schum., Cedrela odorata L., Alseis floribunda Schott, Caesalpinia leiostachya (Benth.) Ducke e 


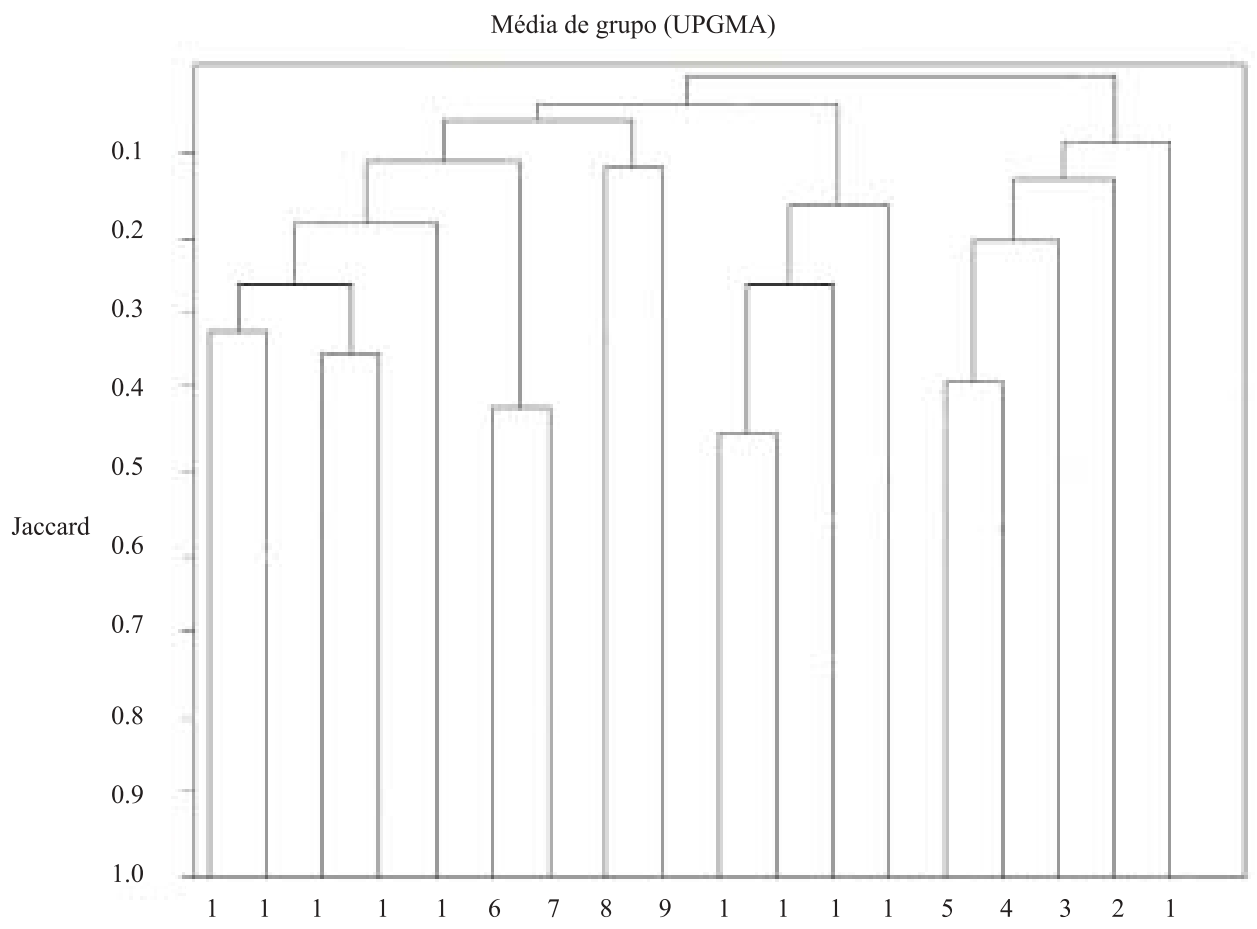

Figura 1. Similaridade florística entre a área de estudo e outras formações vegetacionais. Floresta Ombrófila das Terras Baixas - Mata Atlântica (1 - Lins e Silva 1996; 2 - Siqueira et al. 2001; 3 - Guedes 1998; 4/5 - Barbosa 1996); Floresta Estacional de Altitude - Brejo de Altitude (6/7 - Ferraz 1994; 8 - Correia 1996; 9 - Moura 1997); Vegetação Caducifólia Espinhosa - Caatinga (10/11 - Rodal 1992; $12 / 13$ - Ferraz 1994); 14 - este trabalho; Vegetação Caducifólia não Espinhosa - Carrasco (15/16/17 - Araújo et al. 1998a); e transição Caatinga - Carrasco (18 - Oliveira et al. 1997).

Randia armata (Sw.) DC. Com exceção da primeira espécie, as demais são pouco importantes na estrutura da vegetação em Caruaru, como será visto na seção seguinte; e, como citado anteriormente, são espécies presentes em áreas mais úmidas de VCE ou mesmo em Florestas Estacionais.

Fisionomia e estrutura - A vegetação da área amostrada em Caruaru apresentou fisionomia semelhante à da maioria das áreas da VCE. A densidade total, 3.810 indivíduos ha ${ }^{-1}$, ficou na parte superior da faixa referida para outras áreas da VCE, amostradas com os mesmos critérios de inclusão de plantas (Tab. 3). Naturalmente, trabalhos com critérios de inclusão mais e menos abrangentes têm encontrado densidades maiores e menores, respectivamente
(Sampaio 1996). Considerando apenas as áreas amostradas com os mesmos critérios, há ainda uma variação ampla. É provável que parte desta variação esteja ligada à disponibilidade hídrica, mas não existem estimativas desta disponibilidade nas áreas de vegetação nativa do semi-árido nordestino. A precipitação total anual (Tab. 1) não explica as variações em densidade dos levantamentos de VCE, que por vezes ocorrem em áreas muito próximas; mas, a disponibilidade hídrica envolve outras variáveis, como a distribuição da chuva ao longo do ano e a retenção de água no solo. Também podem contribuir para a variação em densidade as diferenças de manejo das áreas. Apesar de todas terem sido escolhidas pela ausência de sinais de corte de árvores e pelo histórico oral de preservação, umas eram usadas para pecuária 
Tabela 3. Número de indivíduos por hectare e por classes de diâmetro e altura das plantas, em diferentes levantamentos do semi-árido nordestino.

\begin{tabular}{|c|c|c|c|c|c|}
\hline \multirow[t]{2}{*}{ Município } & \multicolumn{3}{|c|}{ Diâmetro $(\mathrm{cm})$} & \multirow{2}{*}{$\begin{array}{c}\text { Altura }(\mathrm{m}) \\
>8\end{array}$} & \multirow[t]{2}{*}{ Referência } \\
\hline & $3-12$ & $>12-27$ & $>27$ & & \\
\hline \multicolumn{6}{|c|}{ Vegetação Caducifólia Espinhosa (VCE) } \\
\hline Caruaru & 2.802 & 305 & 69 & 552 & este trabalho \\
\hline Custódia & 2.128 & 564 & 89 & 238 & Araújo et al. (1995) \\
\hline Custódia & 1.596 & 272 & 60 & 212 & Rodal (1992) \\
\hline Floresta & 1.552 & 268 & 56 & 56 & Rodal (1992) \\
\hline Floresta & 1.872 & 268 & 32 & 28 & Rodal (1992) \\
\hline Custódia & 748 & 176 & 156 & 24 & Rodal (1992) \\
\hline Serra Talhada & 2.800 & 610 & 180 & 50 & Ferraz (1994) \\
\hline Serra Talhada & 3.025 & 410 & 120 & 85 & Ferraz (1994) \\
\hline Floresta & 3.415 & 364 & 67 & 0 & Araújo et al. (1995) \\
\hline Floresta & 1.763 & 319 & 61 & 0 & Araújo et al. (1995) \\
\hline Média & 2.170 & 356 & 89 & 124 & \\
\hline \multicolumn{6}{|c|}{ Vegetação Caducifólia Não Espinhosa (VCNE) } \\
\hline Novo Oriente & 5.266 & 443 & 16 & 576 & Araújo et al.(1998a) \\
\hline Novo Oriente & 6.325 & 272 & 4 & 528 & Araújo et al.(1998a) \\
\hline Novo Oriente & 5.844 & 104 & 4 & 4 & Araújo et al.(1998a) \\
\hline Ubajara & 3.964 & 502 & 13 & 31 & Araújo \& Martins (1999) \\
\hline Média & $5.350 * *$ & $330^{\mathrm{NS}}$ & $9 * *$ & $285^{\mathrm{NS}}$ & \\
\hline \multicolumn{6}{|c|}{ Vegetação de transição VCE - VCNE } \\
\hline Padre Marcos & 4.124 & 444 & 49 & 9 & Oliveira et al. (1997) \\
\hline \multicolumn{6}{|c|}{ Floresta Estacional de Altitude (FEA) } \\
\hline Pesqueira & 3.417 & 834 & 294 & 1.017 & Correia (1996) \\
\hline Jataúba & 3.583 & 667 & 266 & 521 & Moura (1997) \\
\hline Triunfo & 2.290 & 590 & 170 & 510 & Ferraz (1994) \\
\hline Triunfo & 5.510 & 880 & 145 & 370 & Ferraz (1994) \\
\hline Média & $3.700 * *$ & $743 * *$ & $218 * *$ & $604 * *$ & \\
\hline
\end{tabular}

**, * e NS significam diferenças significativas a $1 \%$ e $5 \%$ e não significativa, pelo teste de $\mathrm{t}$ com amostras independentes, em comparação com VCE.

e outras não, como a área de Caruaru.

A área basal total na vegetação de Agreste, em Caruaru $\left(24,9 \mathrm{~m}^{2} \mathrm{ha}^{-1}\right)$, ficou na parte inferior da faixa $\left(15,6\right.$ a $\left.52,4 \mathrm{~m}^{2} \mathrm{ha}^{-1}\right)$ referida para áreas da VCE, amostradas com os mesmos critérios (Sampaio 1996). Isto significa que, comparada a áreas da VCE, a área estudada tem grande quantidade de indivíduos de pequenos diâmetros. Realmente, o número de indivíduos até $12 \mathrm{~cm}$ de diâmetro foi o terceiro mais alto entre as 10 áreas típicas da VCE para as quais se têm dados (Tab. 3). Já os números de indivíduos de $>12$ a $27 \mathrm{~cm}$ e $>27 \mathrm{~cm}$ de diâmetro foram superados pelos de
5 e 6 das 9 outras áreas, respectivamente, e o diâmetro máximo $(47 \mathrm{~cm})$ foi superado pelo de 7 das 9 áreas.

Se a vegetação em Caruaru não se caracterizou por número alto de plantas com grandes diâmetros de caule, o número de plantas altas (> 8m) foi maior que os de todas as áreas da VCE medidas com os mesmos critérios (Tab. 3). A altura máxima atingida (19m) também foi maior que as das áreas da VCE. Assim, a fisionomia da vegetação de Agreste, na área estudada, parece caracterizar-se por densidade grande de plantas que podem atingir 
porte alto, embora não tenham caules grossos. Esta conclusão merece a ressalva de que não há certeza se a área vem sendo preservada há tempo suficiente para que as plantas tenham atingido seu porte máximo. Como quase todas as áreas preservadas do nordeste, não há registros precisos de sua história e a tradição oral apenas aponta a ausência de corte raso há pelo menos 30 anos.

Tendo em conta esta limitação e a carência de dados para outras formações vegetais do semi-árido nordestino, as áreas da Vegetação Caducifólia Espinhosa tiveram densidades menores que as encontradas na Vegetação Caducifólia Não Espinhosa, principalmente de plantas de pequeno porte, possuindo mais indivíduos com caules grossos (Tab. 3). Não há distinção clara quanto ao número de indivíduos com mais de $8 \mathrm{~m}$ de altura, com variação grande nas duas formações. As diferenças na fisionomia podem estar ligadas ao tipo de solo em que se estabelecem, predominando na VCE os solos rasos, de textura média ou argilosa e de boa fertilidade e na VCNE os solos profundos, arenosos e de baixa fertilidade. A vegetação em Padre Marcos, definida como de transição VCE - VCNE, pela sua posição geográfica e pela sua flora (Oliveira et al. 1997), também parece intermediária quanto à densidade e distribuição de diâmetros e alturas e crescia em solo semelhante aos de VCNE.

Todas as áreas da VCE amostradas, com exceção da área de Agreste, em Caruaru, tinham menor número de plantas com altura superior a $8 \mathrm{~m}$ que as Florestas Estacionais de Altitude do semi-árido e quase todas menor número de plantas de qualquer diâmetro, mas principalmente de diâmetro superior a $42 \mathrm{~cm}$ (Tab. 3). Todas as médias das FEA foram significativamente maiores que as de VCE. Como a principal diferença em condições de crescimento entre VCE e FEA é a maior disponibilidade hídrica destas, a falta de água parece ser o fator limitando o porte das plantas de caatinga. Neste caso, a área de Caruaru parece ter melhor condição que as das áreas da VCE, ainda que sua precipitação anual não seja maior que a de muitas delas (Tab. 1) e a capacidade de armazenamento de água no solo não seja elevada. É possível que a distribuição da chuva ao longo do ano seja mais favorável nesta área de Agreste, incluindo o efeito de uma estação de chuvas deslocada para o outono inverno, em contraste com as áreas sertanejas, de verão - outono.

A proporção de plantas mortas ainda em pé na área de estudo $(1,9 \%)$ foi uma das mais baixas já registradas na VCE e em outros tipos de vegetação nordestinos (Sampaio 1996). Como dado isolado no tempo, não permite maiores conclusões sobre a dinâmica da comunidade. Poderia ser um indicativo de baixa taxa de mortalidade ou de queda relativamente rápida das árvores e arbustos mortos. Para esta queda poderia contribuir o relevo acidentado e o solo raso.

$\mathrm{O}$ índice de diversidade na vegetação em Caruaru (3,09 nats indivíduo $\left.{ }^{-1}\right)$ foi maior que o da maioria das áreas da VCE (Sampaio 1996). Áreas com índices semelhantes foram reportadas apenas em Sergipe (Souza 1983; Fonseca 1991) e eram tidas como tendo condições hídricas mais favoráveis que a maior parte do semi-árido. Áreas das Florestas Estacionais de Altitude também tiveram índices semelhantes e até mais altos que os de Caruaru (Ferraz 1994; Correia 1996; Moura 1997). O fato de incluir, além de espécies típicas da VCE, espécies que são geralmente encontradas em áreas mais mésicas, contribuiu para uma flora mais rica que a maioria das áreas da VCE e o número de espécies tem peso grande no cálculo do índice.

As espécies de maior importância na área estudada (Tab. 4) também têm sido relacionadas entre as mais importantes em outros levantamentos em áreas da VCE (Sampaio 1996), confirmando as semelhanças nesta formação. A soma da proporção das três espécies de maior IVI, em relação ao IVI total 
Tabela 4. Parâmetros fitossociológicos das espécies amostradas na Estação Experimental da IPA, em Caruaru, Pernambuco, ordenados decrescentemente pelo IVI. DA - densidade absoluta (indivíduos ha-1); FA - frequiência absoluta (\%); ABA área basal absoluta $\left(\mathrm{m}^{2} \mathrm{ha}^{-1}\right)$; DR - densidade relativa (\%); FR - freqüência relativa (\%); ABR - área basal relativa (\%); IVI - índice de valor de importância.

\begin{tabular}{|c|c|c|c|c|c|c|c|}
\hline Espécie & DA & FA & $\mathrm{ABA}$ & DR & FR & $\mathrm{ABR}$ & IVI \\
\hline Caesalpinia pyramidalis & 513,3 & 96,7 & 2,3792 & 13,47 & 5,63 & 15,91 & 35,01 \\
\hline Schinopsis brasiliensis & 85,0 & 63,3 & 2,6339 & 2,23 & 3,68 & 17,62 & 23,53 \\
\hline Solanum sp. 1 & 486,7 & 93,3 & 0,4789 & 12,77 & 5,43 & 3,20 & 21,40 \\
\hline Bauhinia cheilantha & 408,3 & 76,7 & 0,6893 & 10,72 & 4,46 & 4,61 & 19,79 \\
\hline Maprounea cf. guianensis & 311,7 & 60,0 & 0,5208 & 8,18 & 3,49 & 3,48 & 15,15 \\
\hline Anadenanthera colubrina & 81,67 & 56,7 & 1,3312 & 2,14 & 3,29 & 8,90 & 14,34 \\
\hline Eugenia uvalha & 223,3 & 86,7 & 0,4909 & 5,86 & 5,04 & 2,46 & 14,18 \\
\hline Mimosa malacocentra & 233,3 & 86,7 & 0,3981 & 6,12 & 5,04 & 2,66 & 13,83 \\
\hline Piptadenia stipulacea & 136,7 & 80,0 & 0,3711 & 3,59 & 4,65 & 2,48 & 10,72 \\
\hline Commiphora leptophloeos & 58,3 & 56,7 & 0,8791 & 1,53 & 3,29 & 5,88 & 10,71 \\
\hline Pithecelobium parviflorum & 125,0 & 66,7 & 0,4958 & 3,28 & 3,88 & 3,32 & 10,47 \\
\hline Ptilochaeta bahiensis & 176,7 & 76,7 & 0,1927 & 4,64 & 4,46 & 1,29 & 10,38 \\
\hline Pilosocereus pachicladus & 81,7 & 80,0 & 0,5363 & 2,14 & 4,65 & 3,59 & 10,38 \\
\hline Sapium lanceolatum & 61,7 & 53,3 & 0,7410 & 1,62 & 3,10 & 4,96 & 9,68 \\
\hline Myracrodruon urundeuva & 60,0 & 46,7 & 0,5512 & 1,57 & 3,69 & 2,71 & 7,97 \\
\hline Croton sonderianus & 130,0 & 26,7 & 0,2705 & 3,41 & 1,55 & 1,81 & 6,77 \\
\hline Cereus jamacaru & 41,7 & 53,3 & 0,1548 & 1,09 & 3,10 & 1,51 & 5,23 \\
\hline Capparis flexuosa & 61,7 & 40,0 & 0,1364 & 1,62 & 2,33 & 0,91 & 4,86 \\
\hline Cordia sp. & 53,3 & 26,7 & 0,1137 & 1,40 & 1,55 & 0,76 & 3,71 \\
\hline Manihot dichotoma & 23,3 & 33,3 & 0,1185 & 0,61 & 1,94 & 0,79 & 3,34 \\
\hline Eugenia sp. 2 & 38,3 & 26,7 & 0,0694 & 1,00 & 1,55 & 0,66 & 3,04 \\
\hline Acacia paniculata & 28,3 & 33,3 & 0,0328 & 0,74 & 1,94 & 0,22 & 2,90 \\
\hline Clusia paralicola & 18,3 & 6,70 & 0,2523 & 0,48 & 0,39 & 1,69 & 2,56 \\
\hline Guapira noxia & 18,3 & 26,7 & 0,0341 & 0,48 & 1,55 & 0,23 & 2,26 \\
\hline Tournefortia rubicunda & 23,3 & 26,7 & 0,0144 & 0,61 & 1,55 & 0,10 & 2,26 \\
\hline Croton micans & 45,0 & 10,0 & 0,0593 & 1,18 & 0,58 & 0,40 & 2,16 \\
\hline Capparis jacobinae & 26,7 & 20,0 & 0,0423 & 0,70 & 1,16 & 0,28 & 2,15 \\
\hline Lippia sp. & 18,3 & 26,7 & 0,0143 & 0,48 & 1,55 & 0,10 & 2,13 \\
\hline Jatropha mollissima & 16,7 & 20,0 & 0,0185 & 0,44 & 1,16 & 0,12 & 1,72 \\
\hline Chorisia glaziovii & 6,7 & 13,3 & 0,1037 & 0,17 & 0,78 & 0,69 & 1,64 \\
\hline Lantana camara & 15,0 & 16,7 & 0,0182 & 0,39 & 0,97 & 0,12 & 1,48 \\
\hline Opuntia brasiliensis & 10,0 & 10,0 & 0,0766 & 0,26 & 0,58 & 0,51 & 1,36 \\
\hline Helicteris macropetala & 11,7 & 10,0 & 0,0659 & 0,31 & 0,58 & 0,44 & 1,33 \\
\hline Allophyllus quercifolius & 8,33 & 16,7 & 0,0165 & 0,22 & 0,97 & 0,11 & 1,30 \\
\hline Myrtaceae 1 & 11,7 & 10,0 & 0,0555 & 0,31 & 0,58 & 0,37 & 1,26 \\
\hline Coutarea hexandra & 8,3 & 13,3 & 0,0338 & 0,22 & 0,78 & 0,22 & 1,22 \\
\hline Acacia glomerosa & 11,7 & 10,0 & 0,0408 & 0,31 & 0,58 & 0,27 & 1,16 \\
\hline Solanum sp. 2 & 11,7 & 13,3 & 0,0112 & 0,31 & 0,78 & 0,07 & 1,16 \\
\hline Cedrela odorata & 1,7 & 3,30 & 0,0804 & 0,04 & 0,19 & 0,54 & 0,78 \\
\hline Não identificada 3 & 5,0 & 10,0 & 0,0087 & 0,13 & 0,58 & 0,04 & 0,77 \\
\hline Croton sp. & 5,0 & 10,0 & 0,0067 & 0,13 & 0,58 & 0,04 & 0,76 \\
\hline Mimosa sp. & 5,0 & 6,70 & 0,0271 & 0,13 & 0,39 & 0,18 & 0,70 \\
\hline Cordia globosa & 8,3 & 6,70 & 0,0134 & 0,22 & 0,39 & 0,09 & 0,70 \\
\hline Maytenus rigida & 6,7 & 6,70 & 0,0100 & 0,17 & 0,39 & 0,07 & 0,63 \\
\hline Ziziphus joazeiro & 3,3 & 6,70 & 0,0156 & 0,09 & 0,39 & 0,10 & 0,58 \\
\hline Alseis floribunda & 3,3 & 6,70 & 0,0035 & 0,09 & 0,39 & 0,02 & 0,50 \\
\hline Caesalpinia leiostachya & 1,7 & 3,30 & 0,0380 & 0,04 & 0,19 & 0,25 & 0,49 \\
\hline
\end{tabular}


Tabela 4 (continuação)

\begin{tabular}{lccccccc}
\hline Espécie & DA & FA & ABA & DR & FR & ABR & IVI \\
\hline Acalypha multicaulis & 3,3 & 6,70 & 0,0017 & 0,09 & 0,39 & 0,01 & 0,49 \\
Casearia sp. & 5,0 & 3,30 & 0,0045 & 0,13 & 0,19 & 0,03 & 0,36 \\
Erythroxylum subrotundum & 3,3 & 3,30 & 0,0070 & 0,09 & 0,19 & 0,05 & 0,33 \\
Erythroxylum sp. & 3,3 & 3,30 & 0,0032 & 0,09 & 0,19 & 0,02 & 0,30 \\
Cnidoscolus urens & 3,3 & 3,30 & 0,0020 & 0,09 & 0,19 & 0,01 & 0,29 \\
Eugenia sp. 1 & 1,7 & 3,30 & 0,0049 & 0,04 & 0,19 & 0,03 & 0,27 \\
Randia armata & 1,7 & 3,30 & 0,0020 & 0,04 & 0,19 & 0,01 & 0,25 \\
Croton conduplicatus & 1,7 & 3,30 & 0,0007 & 0,04 & 0,19 & 0,00 & 0,24 \\
\hline
\end{tabular}

(27\%) foi menor que na maioria das áreas da $\mathrm{VCE}$, que geralmente ultrapassa $30 \%$. Isto pode ser reflexo de melhor condição de crescimento e contribui para a maior equabilidade na comunidade. Em outras formações de áreas mais mésicas, incluindo as Florestas Estacionais de Altitude, esta proporção das mais importantes tende a ser ainda mais baixa (Correia 1996; Moura 1997).

A espécie de maior importância, Caesalpinia pyramidalis Tul., é a que aparece mais freqüentemente no topo das listas de estudos de caatinga (Sampaio 1996). Sua densidade e sua área basal relativa tiveram valores próximos e sua frequiência absoluta alta indica que a espécie não só era abundante, mas com boa distribuição no local estudado. A segunda colocada, Schinopsis brasiliensis Engler, é uma das arbóreas de grande porte mais comuns em VCE e seus valores de área basal e densidade correspondem ao padrão em outras áreas: a maior área basal, mas colocação baixa da densidade relativa. Padrão oposto tiveram as três seguintes na colocação do IVI (Solanum sp.1, Bauhinia cheilantha (Bong.) Steud. e Maprounea cf. guianensis Aublet), espécies de porte pequeno, com densidades relativas altas e baixas áreas basais relativas. M. guianensis não se tem destacado como importante em locais da VCE. A sexta espécie em IVI, Anadenanthera colubrina (Vell.) Brenam, é outra das arbóreas de grande porte da VCE do semi-árido nordestino. Sua densidade e sua freqüência foram pouco inferiores às de S. brasiliensis, mas o porte das plantas foi menor, refletido numa área basal relativa de cerca da metade da desta última. A sétima colocada, Eugenia uvalha Camb., é espécie geralmente ausente em levantamentos da VCE e que pode ser característica dos ambientes menos secos do semi-árido, como o do Agreste ou serras e chapadas do sertão.

As espécies seguintes em IVI ocorrem em várias áreas da VCE, destacando-se as outras das arbóreas de grande porte comuns em muitas áreas (Commiphora leptophloeos (Mart.) Gillet, $10^{a}$ colocação, e Myracrodruon urundeuva Allemão, 15 a colocação). Espécies arbóreas pouco comuns em áreas da VCE e mais encontradas em Florestas Estacionais tiveram colocações bem mais baixas: Clusia paralicola G. Mariz, 23 ${ }^{\mathrm{a}}$; Cedrela odorata L., 39 ${ }^{\mathrm{a}}$; Alseis floribunda Schott, 46a ; e Randia armata (Sw.) DC., penúltima. Estas colocações foram em faixa semelhante às de outras arbóreas típicas da VCE mas que raramente ocupam posições de destaque: Chorisia glaziovii (O. Kuntze) E. Santos, 30'; Maytenus rigida Mart., $44^{\mathrm{a}}$; e Ziziphus joazeiro Mart., 45 ${ }^{\mathrm{a}}$. Por outro lado, quase todas as espécies de maior IVI nas Florestas Estacionais de Altitude (Correia 1996; Moura 1997) e na Vegetação Caducifólia Não Espinhosa (Araújo et al. 1998a) não estavam presentes na área de Caruaru. Assim, tanto a estrutura quanto a florística permitem enquadrar a vegetação de Agreste, em Caruaru, como 
Vegetação Caducifólia Espinhosa e podem, quando consideradas em conjunto, diferenciá-la das Florestas Estacionais de Altitude (Brejos) e da Vegetação Caducifólia Não Espinhosa (Carrasco) do Nordeste.

\section{Referências bibliográficas}

Albuquerque, S. G.; Soares, J. G. G. \& Araújo Filho, J. A. 1982. Densidade de espécies arbustivas em vegetação de caatinga. Petrolina, EMBRAPACPATSA. (Pesquisa em andamento, 16).

Alcoforado-Filho, F. G. 1993. Composição florística e fitossociologia de uma área de caatinga arbórea no município de Caruaru-PE. Dissertação de Mestrado. Universidade Federal Rural de Pernambuco, Recife.

Araújo, E. L.; Sampaio, E. V. S. B. \& Rodal, M. J. N. 1995. Composição florística e fitossociológica de três áreas de Caatinga de Pernambuco. Revista Brasileira de Biologia 55(4): 595-607.

Araújo, F. S. \& Martins, F. R. 1999. Variações estruturais e florísticas do Carrasco no planalto da Ibiapaba, estado do Ceará. São Paulo, Acta Botanica Brasilica 13(1): 1-14.

Araújo, F. S.; Sampaio, E. V. S. B.; Rodal, M. J. N. \& Figueiredo, M. A. 1998a. Organização comunitária do componente lenhoso de três áreas de carrasco em Novo Oriente - CE. Revista Brasileira de Biologia 58(1): 85-95.

Araújo, F. S.; Sampaio, E. V. S. B.; Figueiredo, M. A.; Rodal, M. J. N. \& Fernandes, A.G. 1998b. Composição florística da vegetação de carrasco, Novo Oriente - CE. Revista Brasileira de Botânica 21(2): 15-26.

Araújo, G. M. \& Haridasan, M. 1997. Estrutura fitossociológica de duas matas mesófilas semidecíduas em Uberlândia, Triângulo Mineiro. Naturalia 22: 115-129.

Barbosa, M. R. V. 1996. Estudo florístico e fitossociológico da Mata do Buraquinho, remanescente de mata atlântica em João Pessoa, PB. Tese de Doutorado. Universidade Estadual de Campinas, Campinas.

Bigarella, J. J.; Andrade-Lima, D. \& Riehs, P. J. 1975. Considerações a respeito das mudanças paleoambientais na distribuição de vegetais e animais no Brasil. Anais da Academia Brasileira de Ciências 47: 411-467 (suplemento).

Bridson, D. \& Forman, L. 1992. The herbarium handbook. Royal Botanic Gardens, London.
Correia, M. S. 1996. Estrutura da vegetação da mata serrana em um brejo de altitude em Pesqueira PE. Dissertação de Mestrado. Universidade Federal de Pernambuco. Recife.

EMBRAPA 1997. Manual de métodos de análise de solos. EMBRAPA, Brasília.

Emperaire, L. 1985. Végétation de l'État du Piaui, Brésil. Societé de Biogéographie 60(4): 151-163.

Fernandes, A. G. 1996. Fitogeografia do semi-árido. p. 215-219. Anais da $4^{\mathbf{a}}$ Reunião Especial da Sociedade para o Progresso da Ciência. SBPC. Feira de Santana.

Ferraz, E. M. N. 1994. Variação florístico-vegetacional na região do vale do Pajeú, Pernambuco. Dissertação de Mestrado. Universidade Federal Rural de Pernambuco, Recife.

Ferraz, E. M. N.; Rodal, M. J. N.; Sampaio, E. V. S. B. \& Pereira, R. C. A. 1998. Composição florística em trechos de vegetação de caatinga e brejo de altitude na região do Vale do Pajeú, Pernambuco. Revista Brasileira de Botânica 21(1): 7-15.

Figueirêdo, L. S.; Rodal, M. J. N. \& Melo, A. L. 2000. Florística e fitossociologia de uma área de vgetação caducifólia espinhosa no município de Buíque - Pernambuco. Naturalia 25: 205-224.

Fonseca, M. R. 1991. Análise da vegetação arbustivoarbórea da caatinga hiperxerófila do noroeste do estado de Sergipe. Tese de Doutorado. Universidade Estadual de Campinas, Campinas.

Grossman, D. H.; Faber-Langendoen, D.; Weakley, A. S.; Anderson, M.; Bourgeron, P.; Crawford, R.; Goodin, K.; Landaal, S.; Metzler, K.; Patterson, K.; Pyne, M.; Reid, M. \& Sneddon. L. 1998. International Classification of Ecological Communities: Terrestrial vegetation of the United States. v. Arlington: The Nature Conservancy.

Guedes, M. L. S. 1998. A vegetação fanerogâmica da Reserva Ecológica de Dois Irmãos, Pp. 157-172. In: I. C. Machado, A. V. Lopes \& K. C. Porto (eds.). Reserva Ecológica de Dois Irmãos: estudos em um remanescente de Mata Atlântica em área urbana (Recife - Pernambuco - Brasil). Editora Universitária, Recife.

IBGE. 1992. Manual Técnico da Vegetação Brasileira. IBGE. Rio de Janeiro. (Série Manuais Técnicos em Geociências, n. 1).

Kent, M. \& Coker, P. 1992. Vegetation description and analyses: a pratical approach. John Wiley \& Sons, London.

Kozlowski, T. T.; Kramer, P. J. \& Pallardy, S. G. 1991. Physiological ecology of woody plants. Academic Press, New York. 
Larcher, W. 1995. Physiological plant ecology: ecophysiology and stress physiology of functional groups. 3 ed. Springer, Berlin/ London.

Lins e Silva A.C.B. 1996. Florística e fitossociologia do componente arbóreo em um fragmento de mata atlântica na região metropolitana do Recife/PE. Monografia de Graduação. Universidade Federal Rural de Pernambuco, Recife.

Lyra, A. L. R. T. 1982. Efeito do relevo na vegetação de duas áreas do município do Brejo da Madre de Deus (PE). Dissertação de Mestrado. Universidade Federal Rural de Pernambuco, Recife.

Martins, F. R. 1990. Atributos de comunidades vegetais. Quid. 9: 13-17.

Magurran, A. E. 1988. Ecological diversity and its measurement. Princeton University, New Jersey.

Moura, F. B. P. 1997. Fitossociologia de uma mata serrana semidecídua no brejo de Jataúba, Pernambuco, Brasil. Dissertação de Mestrado. Universidade Federal de Pernambuco, Recife.

Mueller-Dumbois, D. \& Ellenberg, H. 1974. Aims and methods of vegetation ecology. John Wiley \& Sons, New York.

Oliveira, M. E. A.; Sampaio, E. V. S. B.; Castro, A. A. J. F. \& Rodal, M. J. N. 1997. Flora e fitossociologia de uma área de transição carrascocaatinga de areia em Padre Marcos, Piauí. Naturalia 22: 131-150.

Prado, D. E. \& Gibbs, P. E. 1993. Patterns of species distributions in the dry seasonal forest of South America. Annals of Missouri Botanical Garden 80: 902-927.

Rizzini, C. T. 1979. Tratado de fitogeografia do Brasil: aspectos sociológicos e florísticos. USP. v 2, São Paulo.

Rodal, M. J. N. 1992. Fitossociologia da vegetação arbustivo-arbórea em quatro áreas de caatinga em Pernambuco. Tese de Doutorado. Universidade Estadual de Campinas, Campinas.

Rodal, M. J. N.; Andrade, K. V. S. A.; Sales, M.F. \& Gomes, A. P. S. 1998. Fitossociologia do componente lenhoso de um refúgio vegetacional no município de Buíque, Pernambuco. Revista Brasileira de Biologia 58(3): 517-526.

Rodal, M. J. N. \& Nascimento, L. M. 2002. Levantamento florístico da floresta serrana da reserva biológica de Serra Negra, microrregião de Itaparica, Pernambuco, Brasil. Acta Botanica Brasilica 16(4): 481-500.
Rodal, M. J. N.; Nascimento, L. M. \& Melo, A. L. 1999. Composição florística de um trecho de vegetação arbustiva caducifólia, no município de Ibimirim, Pernambuco, Brasil. Acta Botanica Brasilica 13(1): 14-29.

Rodal, M. J. N.; Sales, M. F. \& Mayo, S. J. 1998. Florestas serranas de Pernambuco: localização e diversidade dos remanescentes dos brejos de altitude. Imprensa Universitária. Universidade Federal Rural de Pernambuco, Recife.

Rodal, M. J. N.; Sampaio, E. V. S. \& Figueiredo, M. A. 1992. Manual sobre métodos de estudo florístico e fitossociológico - ecossistema caatinga. Sociedade Botânica do Brasil, Brasília.

Sales, M. F.; Mayo, S. J. \& Rodal, M. J. N. 1998. Plantas vasculares das florestas serranas de Pernambuco - Um checklist da flora ameaçada dos brejos de altitude. Imprensa Universitária. Universidade Federal Rural de Pernambuco, Recife.

Sampaio, E. V. S. B. 1996. Fitossociologia. Pp. 203-230. In: Sampaio, E. V. S. B.; Mayo S. J.; Barbosa, M. R. V. (eds.) Pesquisa botânica nordestina: progresso e perspectivas. Sociedade Botânica do Brasil/Seção Regional de Pernambuco, Recife.

Santos, M. F. A. V.; Ribeiro, M. R. \& Sampaio, E. V. S. B. 1992. Semelhanças vegetacionais em sete solos de caatinga. Pesquisa Agropecuária Brasileira 27: 305-314.

Sarmiento, G. 1972. Ecological and floristics convergences between seasonal plant formations of tropical and subtropical South America. Journal of Ecology 60: 367-410.

Shepherd, G. J. 1995. FITOPAC 1. Manual do usuário. Departamento de Botânica. UNICAMP, Campinas.

Shimwell, D. W. 1971. The description and classification of vegetation. University of Washington Press, Seattle.

Siqueira, D. R.; Rodal, M. J. N.; Lins e Silva, A. C. B. \& Melo, A. L. 2001. Physiognomy, structure and floristic in an area of Atlantic Forest in northeast Brazil. Pp. 11-27. In: G. Gottsberger \& S. Liede (eds.). Life forms and Dynamics in Tropical Forests. Gebr. Borntraeger Verlagsbuchhandlung, Berlin-Stuttgart.

Souza, G. V. 1983. Estrutura da vegetação da caatinga hipoxerófila do Estado de Sergipe. Dissertação de Mestrado. Universidade Federal Rural de Pernambuco, Recife. 
Souza, M. J. N.; Martins, M. L. R.; Soares, Z. M. L.; Freitas-Filho, M. R.; Almeida, M. A. G.; Pinheiro, F. S. A.; Sampaio, M. A. B.; Carvalho, G. M. B. S.; Soares, A. M. L.; Gomes, E. C. B. \& Silva, R. A. 1994. Redimensionamento da região semi-árida do Nordeste do Brasil. In: Conferência Nacional e Seminário Latino-Americano de Desertificação. 1994. Fundação Esquel do Brasil, Fortaleza.

Statsoft, G. Inc. 1995. Statistica for Windows (computer program manual). Tulsa.

Tavares, S.; Paiva, F. A. V.; Tavares, E. J. S.; Carvalho, G. H. \& Lima, J. L. S. 1969a. Inventário florestal do Ceará. 1. Estudo preliminar das matas remanescentes do município de Quixadá. Boletim de Recursos Naturais 7(1/4): 93-111.

Tavares, S.; Paiva, F. A. V.; Tavares, E. J. S.; Lima, J. L. S. \& Carvalho, G. H. 1969b. Inventário florestal de Pernambuco. Estudo preliminar das matas remanescentes do município de São José do Belmonte. Boletim de Recursos Naturais 7(1/4): 113-139.
Tavares, S.; Paiva, F. A. V.; Tavares, E. J. S.; Carvalho, G. H. \& Lima, J. L. S. 1970. Inventário florestal de Pernambuco. Estudo preliminar das matas remanescentes do município de Ouricuri, Bodocó, Santa Maria da Boa Vista e Petrolina. Boletim de Recursos Naturais 8(1/2): 149-194.

Tavares, S.; Paiva, F. A. V.; Tavares, E. J. S. \& Lima, J. L. S. 1974a. Inventário florestal do Ceará II. Estudo preliminar das matas remanescentes do município de Tauá. Boletim de Recursos Naturais 12(2): 5-19.

Tavares, S.; Paiva, F. A. V.; Tavares, E. J. S. \& Lima, J. L. S. 1974b. Inventário florestal do Ceará III. Estudo preliminar das matas remanescentes do município de Barbalha. Boletim de Recursos Naturais 12(2): 20-46.

Tavares, S.; Paiva, F. A. V.; Tavares, E. J. S. \& Carvalho, G. H. 1975. Inventário florestal da Paraíba e no Rio Grande do Norte l. Estudo preliminar das matas remanescentes do vale do Piranhas. SUDENE, Recife. (Recursos Naturais, 3). 